\title{
2403. Reduction of satellite flywheel microvibration using rubber shock absorbers
}

\author{
Changcheng Deng ${ }^{1}$, Deqiang $\mathrm{Mu}^{2}$, Yuan $\mathrm{An}^{3}$, Yong Yan ${ }^{4}$ Zongxuan $\mathrm{Li}^{5}$ \\ $1,2,3,4,5$ Institute of Optics, Fine Mechanics and Physics, Chinese Academy of Sciences, \\ Changchun 130033, China \\ ${ }^{1}$ University of Chinese Academy of Sciences, Beijing 100039, China \\ ${ }^{2}$ Changchun University of Technology, Changchun 130012, China \\ ${ }^{1,3}$ Chang Guang Satellite Technology Co., Ltd., Changchun 130000, China \\ ${ }^{1}$ Corresponding author \\ E-mail: ${ }^{1}$ changcheng0211@163.com, ${ }^{2} m u d q @ m a i l . c c u t . e d u . c n,{ }^{3} a n y @ c i o m p . a c . c n,{ }^{4} c c l y y 911 @ 163 . c o m$, \\ 5lizongx@gmail.com
}

Received 27 March 2016; received in revised form 22 October 2016; accepted 25 October 2016 DOI https://doi.org/10.21595/jve.2016.17021

Abstract. Microvibration of flywheels strongly affects the imaging quality of space cameras. A passive vibration method is used in this study to reduce the effect of microvibration. A rubber shock absorber was designed and installed on a satellite. The angular displacement of the second mirror was measured via a fiber optic gyroscopic method. The measured data were imported into MATLAB and analyzed by different methods. The data was plotted as a root-mean-square graph of angular displacement at different speeds along the $\mathrm{x}$-axis, a waterfall plot of the attenuation of force in the $\mathrm{x}$ direction, the vibration spectrum between the frequency and displacement amplitude, and the time domain response of the inverse Fourier transform of the spectrum. The results show that the microvibration of the flywheel causes significant vibration of the imaging system, and that adding a rubber shock absorber can reduce the vibration. The proposed method is a new attempt to analyze microvibration, and can be applied to the engineering design of flywheels.

Keywords: microvibration, vibration isolation, flywheel, rubber shock absorber.

\section{Introduction}

During satellite photography, motion of the moving components of the satellite, such as the reaction flywheel rotation, propulsion jet, attitude control, and solar panel adjustments can cause jitter to the camera, and affect the image quality. The jitter response, also called the microvibration system, cannot be measured and controlled by the attitude control system [1]. Thus, the reaction flywheel rotation has a large impact on image quality, and is a major issue for satellite imaging systems [2].

To reduce the influence of microvibration on the imaging of space cameras, different vibration control technologies have been used [3], namely passive and active vibration isolation, vibration absorption, vibration resistance, and dynamic designs. Among them, the vibration isolation method is the most widely used. The principle of the vibration isolation method is to integrate vibration resilient components, such as springs, rubber mats, and blankets between the object and the supporting surface to isolate the vibration $[4,6]$.

Rubber shock absorbers [7] have the advantage of compact structure, low cost, and ease of manufacturing. They are a commonly used passive vibration isolator, and are used in many different engineering fields. In addition, they have a damping ratio of 0.06-0.1, and can absorb mechanical energy, especially high-frequency mechanical energy. They can be bonded with metal, forming a multilayered structure to bear load, reduce system stiffness, and change its vibration frequency range. A review of the application of rubber shock absorbers in aerospace can be found in [8]. An accurate and efficient finite element modeling and static characteristics analysis of rubber shock absorbers was proposed by Haiting [9-12]. Sjoberg investigated rubber shock absorber dynamic modeling and dynamic characteristics [13], and used the fractional derivative method to establish a numerical model [14].

A rubber shock absorber was used in this study to reduce the effect of microvibration from the 
flywheel. It was placed between the vibration source (flywheel) and the load board [15, 16]. The effects of vibration isolation of rubber shock absorber were measured experimentally. The satellite used in this study was Jilin No. 1 . The parameters of the camera were: focal length $f=8 \mathrm{~mm}, F$ number $F \#=13.3$, and incident light in the $500 \mathrm{~nm}-800 \mathrm{~nm}$ range. The angular displacement of the second mirror was measured by the fiber optic gyro method [17], and then the measured results were imported into the MATLAB program. Different analysis methods were used in the time and frequency domains to obtain relative plots. The experiment is carried out on a real satellite, and the results are reliable, and thus can be used as a reference for engineering design.

\section{The causes of reaction wheel vibration}

Among the many microvibration sources, the microvibration of the flywheel is the most critical. The main cause of flywheel vibration is the flywheel rotor being unbalanced [18], which can be caused by many reasons, such as:

1) Fabrication issues such as uneven material quality, poor manufacturing precision, and structural asymmetry;

2) Assembly and installation issues such as poor installation, eccentricity, and loose parts;

3) Issues that develop during operation of the satellite, such as shaft bending and deformation, and wear, corrosion, fracture, or deposition of the rotating parts.

\section{Methods of vibration isolation of the reaction flywheel}

Owing to the requirement for high reliability in aerospace applications, the passive isolation method is generally used, because it does not require external energy input, the system is always stable, and the isolation effect on high frequency vibration is significant.

Movement of the reaction flywheel will produce vibration, which is mostly high-frequency sinusoidal vibration. The vibration isolation mechanism is equivalent to a high-frequency sinusoidal vibration being conducted to a low-pass filter; therefore, the vibration from the reaction flywheel acting on the satellite is mainly low-frequency sinusoidal vibration.

In a passive vibration isolation scheme, the requirements for safe operation of the carrier and the aircraft can be met by selecting reasonable parameters and layout, such as the diameter, thickness, and distributed diameter of the vibration isolation. To reduce the negative impact of microvibration, the following factors need to be considered:

1) Disturbance characteristics of the microvibration source (reaction flywheel);

2) Satellite structure and the vibration isolation requirements of on-board equipment; and

3) Imaging conditions of the camera in orbit and the integral series of Charge Coupled Device (CCD) Time Delay Integration (TDI)

\section{Rubber shock absorber design}

\subsection{Rubber shock absorber principles}

The rubber shock absorber is a single-axis isolation system with stiffness and viscous damping. The absolute and relative transmissibility curves are shown in Fig. 1 and Fig. 2, respectively. Different curves of the damping ratio, $\zeta$, intersect at point $(\sqrt{2}, 1)$ in Fig. 1 . The absolute transmissibility of force (displacement) is maximum when $\omega=\omega_{n}$, and tends to infinity when $\zeta=0$, when resonance occurs. For a vibration damper to function as an isolation system, the absolute transmissibility of force (displacement) should less than 1 , thus $\omega>\sqrt{2 \omega_{n}}$. The absolute transmissibility $\mu$ decreases as $\omega / \omega_{n}$ increases, which means that the isolation becomes better. However, the ratio of $\omega / \omega_{n}$ cannot be too large. To obtain that case, the isolation system must be designed to be very soft; the system then becomes less stable and can easily be shaken by external disturbances. The relative transmissibility $\mu_{r e l}$ does not change much if $\omega>5 \omega_{n}$ as shown in 
Fig. 2. The ratio ranges are generally from 2.5 to 5 in real situations. With increasing $\zeta$, the absolute transmissibility becomes large and the relative transmissibility becomes small, and thus the effects of isolation are reduced. It is important to select the optimal value of $\zeta$, which ranges from 0.05 to 0.2 . In this range, the resonance amplitude is small, and the effects of isolation are not reduced too much. Rubber is selected as the material if $\zeta$ ranges from 0.1 to 0.2 .

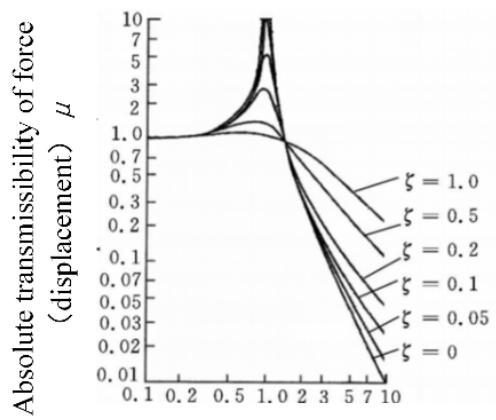

Frequency ratio $\omega / \omega_{n}$

Fig. 1. The absolute transmissibility of a vibration damper with stiffness and viscous damping

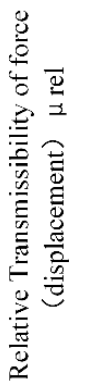

Fig. 2. The relative transmissibility of a vibration damper with stiffness and viscous damping

\subsection{Rubber shock absorber structure}

One flywheel was used in the experiment, which had a mass of $3.585 \mathrm{~kg}$, and was attached to the bracket by four screws (the mass of bracket is $1.11 \mathrm{~kg}$ ). The flywheel was installed on the $y$ load plate and each screw was mounted with two rubber shock absorbers, as shown in Fig. 3.
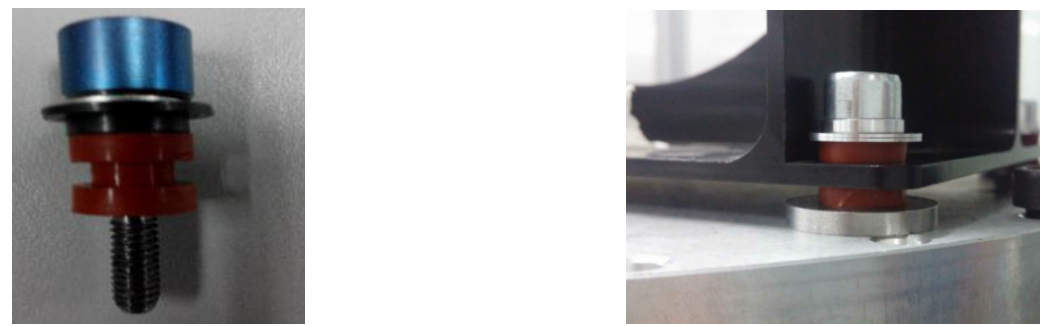

Fig. 3. Photo of rubber shock absorber

The limit static stress $\sigma$ of rubber shock absorber can be calculated as:

$\sigma=\frac{F}{S}$

where $F$ is the static load of the shock absorber and $S$ is the minimum bearing area of the shock absorber. For low damping material, $\sigma$ is $1.8 \mathrm{MPa}$; thus, the minimum area of the shock absorber is $6.39 \mathrm{~mm}^{2}$ with a static load of $11.5 \mathrm{~N}$ according to Eq. (1) [19].

The natural frequency of a shock absorber $f_{n}$ is:

$f_{n}=\frac{1}{2 \pi} \sqrt{\frac{k g}{G}}$,

where $k$ is the dynamic stiffness of the shock absorber, $g$ is gravitational acceleration, and $G$ is the gravity force of the shock absorber. According to technical handbooks on the satellite 
equipment, the vibration frequency ranges from 10 to $2000 \mathrm{~Hz}$. If the vibration frequency meets the condition $f>\sqrt{2} f_{n}$ (supposing its natural frequency $f_{n}=10 \mathrm{~Hz}$ ), then rubber shock absorber damping takes effect, and the total dynamic stiffness is $K=18516.33 \mathrm{~N} / \mathrm{m}$ according to Eq. (2).

The complex shape of the shock absorber should be taken into account, considering the parallel and series connection of components. The total rigidity can be written as:

$k_{c}=k_{c 1}+k_{c 2}+\cdots$,

$\frac{1}{k_{b}}=\frac{1}{k_{b 1}}+\frac{1}{k_{b 2}}+\cdots$,

where $k_{c}$ is a parallel connection and $k_{b}$ is a series connection.

For ease of calculation, the stress-strain relationship of rubber damper is considered to be linear, as:

$K_{S}=\alpha \frac{S E}{h}$,

where $K_{S}$ is the static stiffness of the shock absorber, and $K / K_{S}$ generally equals $1.2-2, S$ is the effective load area of the shock absorber, $E$ is the effective modulus of the shock absorber, $h$ is the height of the shock absorber, and $\alpha$ is a coefficient approaching a value of 1 according to experience from previous experiment.

Letting $K / K_{S}=1.5$, the total stiffness is $K_{S}=12344.22 \mathrm{~N} / \mathrm{m}$. Supposing that shock absorbers uniformly bear the load, the static stiffness of each shock absorber according to Eq. (3) is $K_{S}^{\prime}=3086.05 \mathrm{~N} / \mathrm{m}$. Supposing that the shock absorber is cylindrical, and the diameter is $d$, the values of $d$ and $h$ can be optimized using the equations below [20]. The free-body diagram is shown in Fig. 4. The forces and moments for the flywheel come from the static and dynamic unbalance of the flywheel:

$\operatorname{Min}: \mu_{x}=\left[\frac{1+\left(\frac{\delta \omega}{\pi \omega_{n}}\right)^{2}}{\left(1-\frac{\omega^{2}}{\omega_{n}^{2}}\right)^{2}+\left(\frac{\delta \omega}{\pi \omega_{n}}\right)^{2}}\right]^{1 / 2}$,

where:

$\omega_{n}=\left[\frac{k_{s}}{\frac{m m_{f}}{m+m_{f}}}\right]^{1 / 2}=\left[\frac{\frac{\alpha S E}{h}}{\frac{m m_{f}}{m+m_{f}}}\right]^{1 / 2}=\left[\frac{\alpha E d^{2}}{\frac{4 h m m_{f}}{m+m_{f}}}\right]^{1 / 2}, \quad \delta=\frac{\pi c}{\omega_{n} \frac{m m_{f}}{m+m_{f}}}$,

s.t. $\left\{\begin{array}{l}\sigma_{b}=\frac{M}{W}=\frac{T_{d}+F_{u}\left(h+\frac{l}{2}\right)}{\frac{\pi d^{3}}{32}}=\frac{32\left(T_{d}+F_{u}\left(h+\frac{l}{2}\right)\right)}{\pi d^{3}} \leq\left[\sigma_{b}\right], \\ \tau=\frac{4 F_{s}}{3 \pi r^{2}}=\frac{16 F_{u}}{3 \pi d^{2}} \leq[\tau], \\ 5 \times 2 \pi \leq \omega \leq 50 \times 2 \pi, \\ 2.5 \leq \frac{\omega}{\omega_{n}} \leq 5,\end{array}\right.$

where $\mu_{x}$ is the ratio of the displacement between the flywheel and the foundation, $\sigma_{b}$ is the normal stress of winding due to bending moment, $\tau$ is the shear stress, $M$ is the bending moment, 
$W$ is the section factor of bending resistance, $F_{s}$ is the shear force, $F_{u}$ is the force of static unbalance of the flywheel in the radial direction, $T_{d}$ is the moment of force caused by the flywheel being dynamically unbalanced, $l$ is the length of the flywheel, $\omega$ is the frequency of the excitation, $\omega_{n}$ is the natural frequency of the system, $m$ is the mass of the flywheel and support, $m_{f}$ is the mass of the foundation, $m_{s}$ is the static unbalance mass of flywheel, $m_{d}$ is the dynamic unbalance mass of the flywheel, and $c$ is the viscous damping coefficient. The fmincon function of MATLAB was used for optimization of $d$ and $h$.

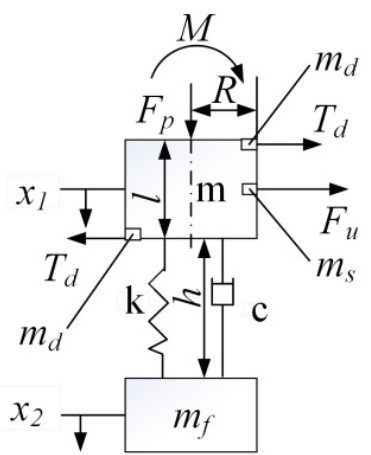

Fig. 4. Isolation model of rubber shock absorber

After several iterations of computation, we obtained the optimized values of the parameters as $d=6.23 \mathrm{~mm}$ and $h=6.11 \mathrm{~mm}$. The rubber shock absorbers were chosen to match these values. There was a threaded hole in the center of the rubber shock absorber. The section area of the rubber shock absorber cannot be less than $S=\pi\left(d^{2}-d_{h}^{2}\right) / 4$ according to Eq. (1), where $d_{h}$ is the diameter of the hole. In addition, the diameter of the flange and the other end of the absorber were determined, and an M5 screw was chosen. The structural dimensions of the rubber shock absorber [21] are shown in Fig. 5. The damping ratio of the rubber shock absorber was 0.1 according to the material and structure. The material was rubber made in institute 703 of the China Aerospace Science and Technology Corporation.
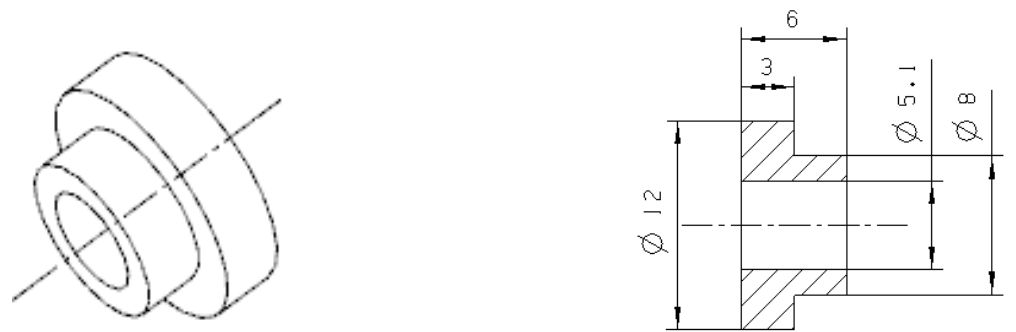

Fig. 5. Structure of rubber shock absorber

\section{Experimental investigation}

The fiber optic gyroscope method was used to measure the angular displacement of the second mirror. The position of the second mirror is shown in Fig. 6.

\subsection{Principle of the fiber optic gyroscope method}

The fiber optic gyroscope method is based on the Sagnac effect in a circular optical system, as shown in Fig. 7.

When a beam of light from a beam splitter is introduced to the two identical optical loops, it forms 2 beams $C_{c w}$ and $C_{c c w}$ that propagate clockwise $(\mathrm{CW})$ and counter-clockwise $(\mathrm{CCW})$, 
respectively. When the loop rotates around its vertical axis, the 2 beams generate a phase difference with a size proportional to the angular velocity of the optical loop.

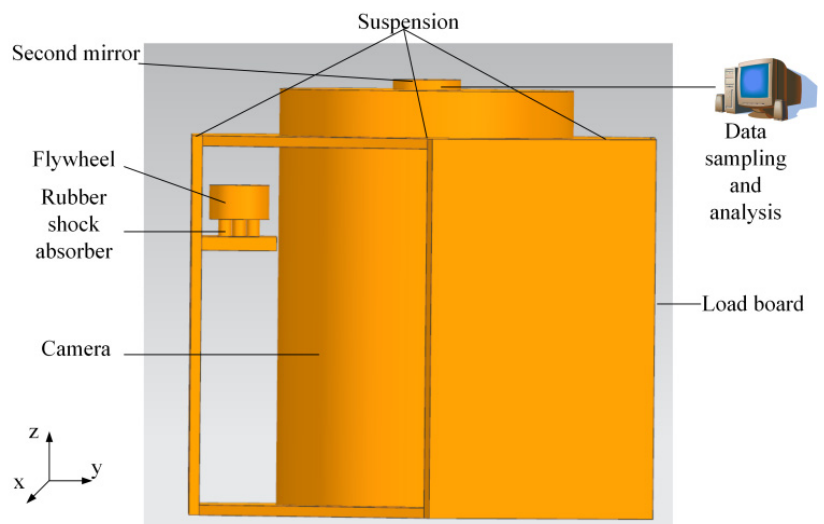

Fig. 6. The schematic diagram of the experimental setup

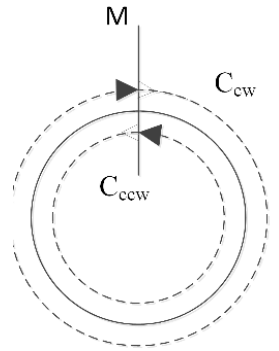

a) Static system

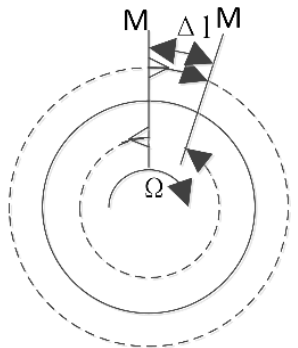

b) Rotated system

Fig. 7. Sagnac effect of an ideal loop system of the light

As shown in Fig. 7(a), the propagation time of the 2 beams is the same without rotation, as shown in Eq. (8):

$t_{c c w}=t_{c w}=\frac{L}{c}=\frac{2 \pi R}{c}$

where $t_{c c w}$ is the propagation time of the counter-clockwise light, $t_{c w}$ is the propagation time of the clockwise light, $L$ is the total length the fiber contained within the ring, $c$ is the speed of the light, and $R$ is the radius of the optical fiber ring.

As shown in Fig. 7(b), $t_{c c w}$ and $t_{c w}$ in a rotated system are:

$t_{c c w}=\frac{2 \pi R}{c-\Omega R}$

$t_{c w}=\frac{2 \pi R}{c+\Omega R}$

where $\Omega$ is the angular velocity.

Because $c^{2}$ is much larger than $(\Omega R)^{2}$, the difference of the propagation time $\Delta t$ is:

$\Delta t=t_{c c w}-t_{c w}=\frac{4 \pi \Omega R^{2}}{c^{2}}$

The optical path difference is: 
$\Delta L=\Delta t \cdot c=\frac{4 \pi R^{2} \Omega}{c}$

The phase difference caused by propagation $\Delta \Phi_{s}$ is:

$\Delta \Phi_{s}=\frac{4 \pi R L}{\lambda_{0} c} \Omega$

where $\lambda_{0}$ is the length of the wave.

The optical path difference caused by rotation of the optical fiber is very small and difficult to measure, thus optical fiber coils containing multiple turns are used to increase the Sagnac effect in real applications. For an optical fiber ring of $N$ turns, the length of the light spreading in the loop is:

$L=N \cdot 2 \pi R$,

and the phase difference for spreading $\Delta \Phi_{S}$ is thus:

$\Delta \Phi_{s}=\frac{4 \pi R L N}{\lambda_{0} c} \Omega$

The phase difference for spreading can be measured by the change of the intensity of the interference light according to the interference principle of the light, thus both the angular displacement and velocity can be obtained.

\subsection{Experimental system}

The experimental equipment included a satellite with a camera, a reaction flywheel, a fiber optic gyro, a data acquisition board, and a computer as shown in Fig. 6.

The vibration source in the experiments is the flywheel. The flywheel rotation axis was parallel to the optical axis. To simulate the real flying conditions of satellite and the approximate real gravity environment in space, the satellite was suspended with a soft sling.

The vibration of the flywheel propagates to the second mirror, and thus the effects of vibration can be measured by the movement of the second mirror. The angular displacement of the second mirror was measured by the fiber optic gyro [22-25], because the angular displacement of the second mirror is the largest of all the mirrors (equivalent to one end of the cantilever beam). The distributions of all the mirrors are shown in Fig. 8. The data results were collected by a data acquisition device and transferred to a computer in real time, in which it was processed using the MATLAB program. The data were analyzed and calculated in the time and frequency domains, and graphs were output in 2 and 3 dimensions, to describe the change of parameters related to the vibration.

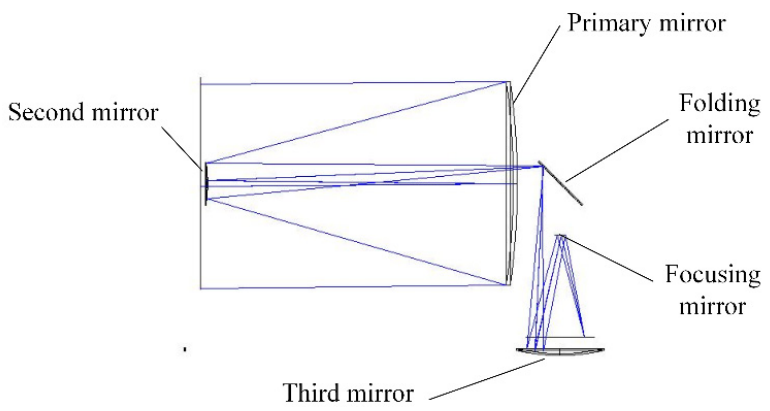

Fig. 8. The light path 
Two experimental setups were constructed, one with a rubber shock absorber, and the other without, and the experimental results were compared.

\subsection{Uncertainty analysis}

The error model [26] of the fiber optic gyro $y=f\left(\theta_{x}, \theta_{y}, \theta_{z}, \Delta t\right)$ ( $y$ is the error function) is:

$$
\left\{\begin{array}{l}
\frac{N_{x}}{K_{x}}=\theta_{x}+E_{x z} \theta_{y}+E_{x y} \theta_{z}+D_{x} \Delta t, \\
\frac{N_{y}}{K_{y}}=E_{y z} \theta_{x}+\theta_{y}+E_{y x} \theta_{z}+D_{y} \Delta t, \\
\frac{N_{z}}{K_{z}}=E_{z y} \theta_{x}+E_{z x} \theta_{y}+\theta_{z}+D_{z} \Delta t,
\end{array}\right.
$$

where $N_{x}, N_{y}$, and $N_{z}$ are the outputs of the fiber optic gyro along the $x-, y-$, and $z$-axes, respectively; $K_{x}, K_{y}$, and $K_{z}$ are scale factors of the fiber optic gyro; $\theta_{x}, \theta_{y}$, and $\theta_{z}$ are inputs of the fiber optic gyro; $E_{x z}$ and $E_{x y}$ are the installation errors of $\theta_{y}$ and $\theta_{z}$, respectively; $E_{y z}$ and $E_{y x}$ are the installation errors of $\theta_{x}$ and $\theta_{z}$, respectively; $E_{z y}$ and $E_{z x}$ are the installation errors of $\theta_{x}$ and $\theta_{y}$, respectively; and $D_{x}, D_{y}$, and $D_{z}$ are round-off errors along the $x-, y-$, and $z$-axes, respectively.

The angular displacement was tested 60,340 times. The uncertainty of a repeated test is calculated by a type A evaluation of uncertainty. The value is the standard deviation divided by the root of the number of tests. The uncertainty of repeated measurement with isolation is $u_{\theta}=\left[\begin{array}{lll}u_{\theta x} & u_{\theta y} & u_{\theta z}\end{array}\right]=\left[\begin{array}{lll}0.5586 & 0.5570 & 0.5525\end{array}\right]$ along $x$-, $y$-, and $z$-axes, respectively:

$u_{\theta}=\frac{\sigma}{\sqrt{n}}=\left[\frac{\sigma_{x}}{\sqrt{n}}, \frac{\sigma_{y}}{\sqrt{n}}, \frac{\sigma_{z}}{\sqrt{n}}\right]$

where $\sigma$ is the standard deviation of angular displacement with isolation, which is given by

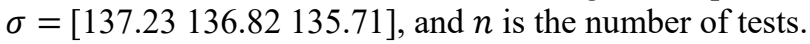

The type B evaluation of uncertainty consists of the uncertainty of the angle, the error of the null position, the error of the stability of the scale factor, and random drift. They should all follow a normal distribution.

The angle error is $\pm 0.001 "$, and the uncertainty is:

$u_{a}=\frac{0.001}{\sqrt{3}}=5.77 e-4$.

The error of null position is \pm 0.0005 ", and the uncertainty is:

$u_{z}=\frac{0.0005}{\sqrt{3}}=2.89 e-4$

The error of the stability of scale factor and random drift is:

$u_{r}=\frac{0.002}{\sqrt{3}}=1.15 e-3$

The combined uncertainty with isolation is: 
$u_{c}=\sqrt{u_{\theta}^{2}+u_{a}^{2}+u_{z}^{2}+u_{r}^{2}}=\left[\begin{array}{lll}u_{c x} & u_{c y} & u_{c z}\end{array}\right]=\left[\begin{array}{lll}0.5586 & 0.5570 & 0.5525\end{array}\right]$,

where:

$u_{c x}=\sqrt{u_{\theta x}^{2}+u_{a}^{2}+u_{z}^{2}+u_{r}^{2}}$

$u_{c y}=\sqrt{u_{\theta y}^{2}+u_{a}^{2}+u_{z}^{2}+u_{r}^{2}}$,

$u_{c z}=\sqrt{u_{\theta Z}^{2}+u_{a}^{2}+u_{z}^{2}+u_{r}^{2}}$.

The resolution of the sample time is $0.002 \mathrm{~s}$ and the distribution follows a normal distribution. Therefore, the uncertainty of the sample time is:

$u_{c t}=\frac{0.002}{2 \sqrt{3}}=5.77 e-4$.

For the output of the fiber optic gyro, the combined uncertainty of the function $f$ with isolation is:

$u_{f}=K \cdot \sqrt{\left(\frac{\partial f}{\partial \theta_{x}}\right)^{2} u_{c x}^{2}+\left(\frac{\partial f}{\partial \theta_{y}}\right)^{2} u_{c y}^{2}+\left(\frac{\partial f}{\partial \theta_{z}}\right)^{2} u_{c z}^{2}+\left(\frac{\partial f}{\partial \Delta t}\right)^{2} u_{c t}^{2}}$,

where:

$u_{f x}=K_{x} \cdot \sqrt{u_{c x}^{2}+E_{x z}^{2} u_{c y}^{2}+E_{x y}^{2} u_{c z}^{2}+D_{x}^{2} u_{c t}^{2}}$,

$u_{f y}=K_{y} \cdot \sqrt{E_{y z}^{2} u_{c x}^{2}+u_{c y}^{2}+E_{y x}^{2} u_{c z}^{2}+D_{y}^{2} u_{c t}^{2}}$,

$u_{f z}=K_{z} \cdot \sqrt{E_{z y}^{2} u_{c x}^{2}+E_{z x}^{2} u_{c y}^{2}+u_{c z}^{2}+D_{z}^{2} u_{c t}^{2}}$,

$E=\left[\begin{array}{ccc}1 & E_{x z} & E_{x y} \\ E_{y z} & 1 & E_{y x} \\ E_{z y} & E_{z x} & 1\end{array}\right]=\left[\begin{array}{ccc}1 & -0.019 & 0.00017 \\ 0.0187 & 1 & -0.0002 \\ -0.000054 & 0.00041 & 1\end{array}\right]$,

$D=\left[\begin{array}{lll}D_{x} & D_{y} & D_{z}\end{array}\right]=\left[\begin{array}{ll}0.0087-0.0183 & 0.003\end{array}\right]$,

$K=\left[\begin{array}{lll}K_{x} & K_{y} & K_{z}\end{array}\right]=\left[\begin{array}{lll}0.9998 & 1.0005 & 1.0007\end{array}\right]$,

$u_{f}=\left[\begin{array}{lll}u_{f x} & u_{f y} & u_{f z}\end{array}\right]=\left[\begin{array}{lll}0.5586 & 0.5574 & 0.5529\end{array}\right]$.

The values of $u_{\theta}^{\prime}$ without isolation are $u_{\theta}^{\prime}=\left[\begin{array}{lll}0.218 & 0.2254 & 0.2243\end{array}\right]$. The values of $u_{f}^{\prime}$ without isolation are $u_{f}^{\prime}=\left[\begin{array}{lll}0.218 & 0.2256 & 0.2245\end{array}\right]$.

\subsection{Experimental process}

A schematic of the experimental setup is shown in Fig. 6. Before the experiment, we needed to check that the bolts were screwed down and the circuit was operating correctly. Then, the flywheel was powered on and the speed of the flywheel increased gradually from 0 to $3000 \mathrm{rpm}$. In this process, the flywheel speed was controlled by the software platform. When the speed reached $300 \mathrm{rpm}$, the fiber optic gyro started to record, and then the speed was increased in $200 \mathrm{rpm}$ steps. When the speed was stable, the angular displacements of the second mirror were 
recorded at the corresponding speed.

\section{Experimental results}

The data were analyzed by different methods. The angular displacement of the second mirror was obtained by the fiber optic gyro, and the data were imported into MATLAB. Then, by analysis in the time domain, the root mean square of angular displacement along the $x$-axis was obtained as shown in Fig. 9. Fourier transforms were performed of the data in Fig. 9 as a waterfall plot. The data was plotted in 3 dimensions to show the relationship between the flywheel speed (motor frequency) and the frequency and amplitude of response, as shown in Fig. 10. The frequency spectrum of the displacement amplitude when the speed of the flywheel was selected is shown in Fig. 11. To isolate the noise and the shake of the sling, the time domain response graph after the inverse Fourier transform of the spectrum was calculated, and is shown in Fig. 12.

The experiment involved two coordinate systems, namely the satellite and flywheel coordinate systems. In the satellite system, the $z$-axis was the optical axis, the $x$-axis was the direction of flight, and the $y$-axis was defined by the right hand coordinate system. In the flywheel coordinate system, the $z$-axis was the rotation axis, and the $x$ - and $y$-axes were defined by the right hand coordinate system. The two systems are parallel and only differ in the origin.

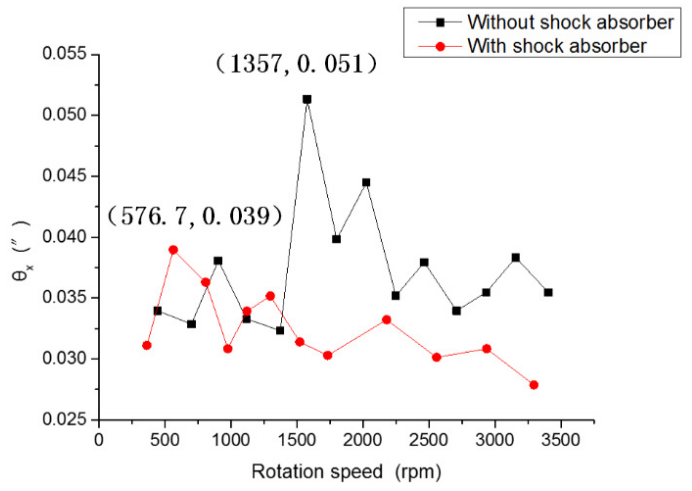

Fig. 9. Root mean square of angular displacement along $x$-axis at different speeds of the second mirror

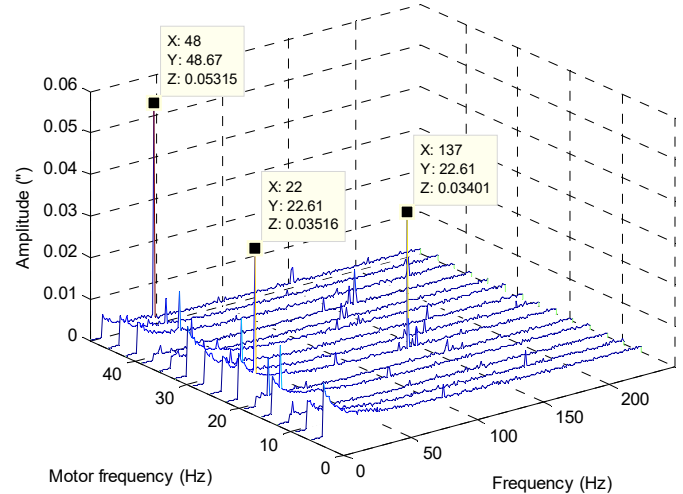

a) Without shock absorber

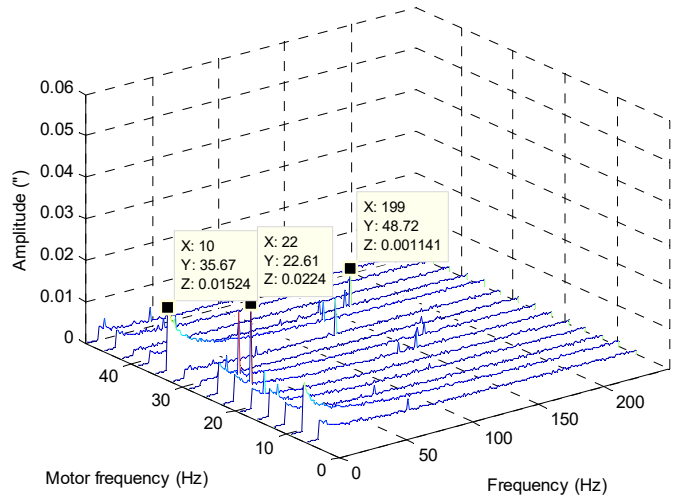

b) With shock absorber

Fig. 10. Waterfall plot of the flywheel speed (motor frequency), frequency, and amplitude of the response

Fig. 9 shows the root mean square of angular displacement along the $x$-axis at different speeds of the second mirror, and shows that the decrease of response at high speed was considerable. However, the angular displacement was larger at low speeds with a rubber shock absorber than without. The reason for this is that the peak lies near the normal modal frequency of the model 
(resonance). The modal frequency with a rubber shock absorber is lower than without. The maximum angular displacement along the $x$-axis with the shock absorber reduced as the speed of the flywheel increased. Including a rubber absorber in the system caused the maximum angular displacement along the $x$-axis to decrease by $23.5 \%$ from 0.051 " to 0.039 ", and the maximum rotation speed changed from $1357 \mathrm{rpm}$ to $576.7 \mathrm{rpm}$. Thus, the rubber shock absorber is useful if the speed is larger than $1300 \mathrm{rpm}$.

The waterfall plot is used to identify the disturbance trends across both frequency and rotational speed in the system. It is obtained by transforming the time domain data into the frequency domain, which is plotted side-by-side in a 3-dimensional diagram. The waterfall plot of the flywheel speed (motor frequency) and frequency and amplitude of the response is shown in Fig. 10.

The results in Fig. 10 show that the peaks were concentrated at low frequencies. The relation between the flywheel speed and the amplitude was unclear. When comparing the situation with and without the rubber shock absorber in Fig. 10, the number and the values of peaks with the rubber shock absorber were less than those without it, for frequencies less than $50 \mathrm{~Hz}$. For frequencies from $100 \mathrm{~Hz}$ to $200 \mathrm{~Hz}$, there were a few peaks without the rubber shock absorber, but a much lower number when the shock absorber was introduced. When the shock absorber was introduced, the largest peak decreased by $58.5 \%$ from $0.053 "$ at $48 \mathrm{~Hz}$ to 0.022 " at $22 \mathrm{~Hz}$. The frequency in the graph was less than $250 \mathrm{~Hz}$ in this model, because the vibration in this range has a significant effect on the quality of the image.

Fig. 11 plots the spectrums of angular displacement, with and without rubber shock absorber, along the $x$-axis. It is shown that with the absorber added, the peak of vibration decreased from $0.04775 "$ at $137.6 \mathrm{~Hz}$ to $0.004644 "$ at $50.8 \mathrm{~Hz}$. It is noted here that the angular displacements are set as 0 for frequencies less than $50 \mathrm{~Hz}$, to avoid the influence of noise and satellite shake. It is shown that with the shock absorber added, the angular displacement decreased as the frequency increased, which means that the effects of isolation were better at high frequency than at low frequency. It is also found that, with shock absorber added, the decrease of the angular displacement becomes slower for frequencies larger than $100 \mathrm{~Hz}$, and the average of the angular displacement maintained its value for frequencies from $150 \mathrm{~Hz}$ to $250 \mathrm{~Hz}$. This means that for the case with the rubber shock absorber, the angular displacement was not dependent on the frequency in the range from $150 \mathrm{~Hz}$ to $250 \mathrm{~Hz}$.

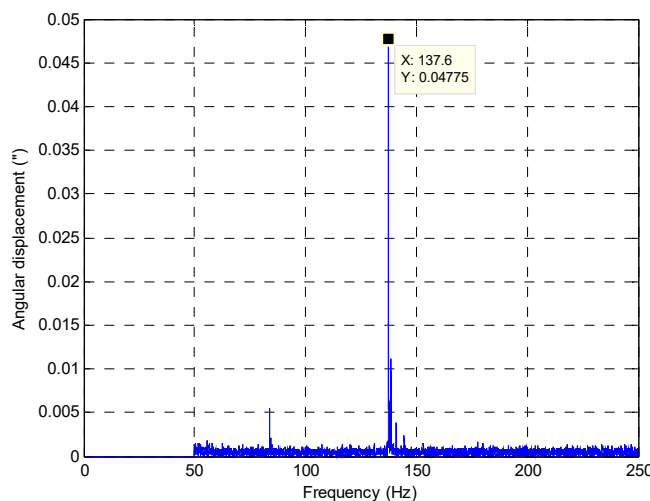

a) Without shock absorber

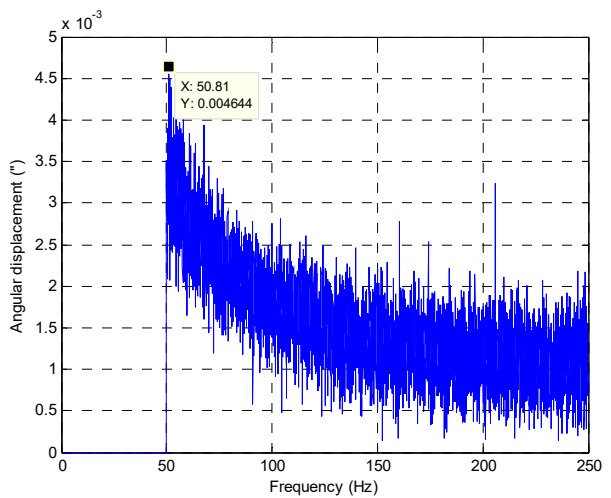

b) With shock absorber

Fig. 11. Angular displacement spectrum of $x$-axis after filtering

The time domain response of vibration by inverse Fourier transform of the $x$-axis spectrum was obtained, and plotted in Fig. 12, for the case without and with the rubber shock absorber. The range of angular displacement changed from larger than 0.15 " to less than 0.1 " with absorber added, and the vibration isolation effect was considerable. 


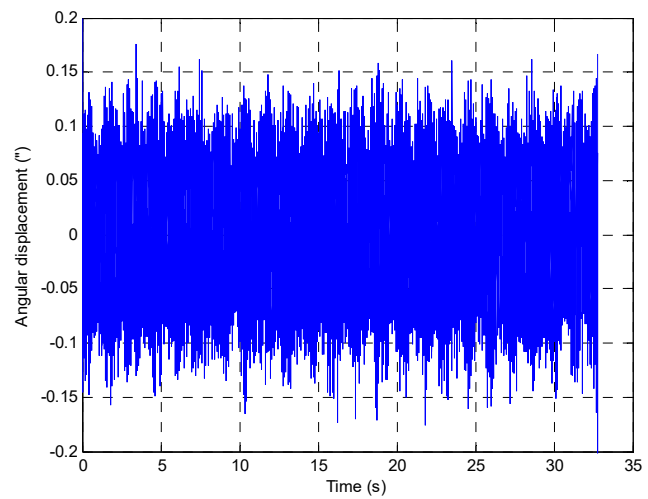

a) Without shock absorber

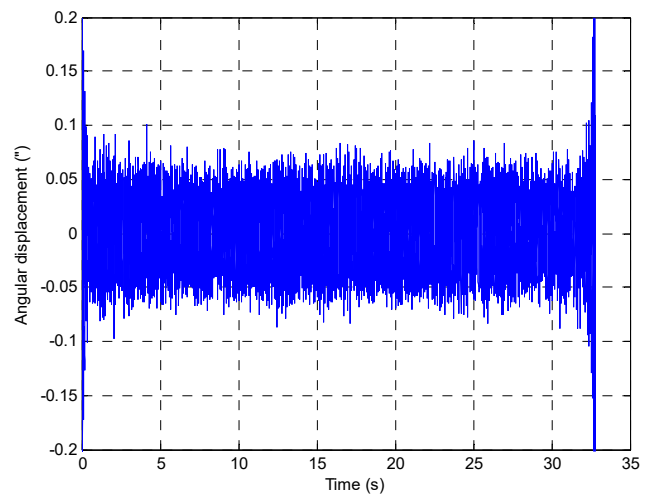

b) With shock absorber

Fig. 12. Response of time domain after the inverse Fourier transform of the $x$-axis spectrum

\section{Conclusions}

The movement of the reaction flywheel is a major source of satellite vibration. Vibration from the flywheel is transferred to the second mirror through the whole satellite, and reduces the quality of images. To reduce the vibration of the flywheel, a rubber shock absorber has been designed and added to the satellite, and experiments performed to quantify the effects of adding the rubber shock absorber. The angular displacement of the second mirror was obtained using a fiber optic gyro. Then, time domain analysis, waterfall plot analysis, and spectrum analysis were performed. The results show that the vibration of flywheel causes considerable vibration of the second mirror, and the vibration is varied with the rotation speed of the flywheel and frequency of the system. Using a rubber shock absorber was found to decrease the vibration significantly, is thus a useful way to improve imaging quality, and should be considered for the engineering design of satellites.

\section{Acknowledgements}

The author would like to thank the Changchun Institute of Optics, Fine Mechanics and Physics and the Chinese Academy of Sciences for assistance with the experiments.

\section{References}

[1] Wei Z., Li D., Luo Q., Jiang J. Modeling and analysis of a flywheel microvibration isolation system for spacecrafts. Advances in Space Research, Vol. 55, Issue 2, 2015, p. 761-777.

[2] Lee D. O., Park G., Han J. H. Experimental study on on-orbit and launch environment vibration isolation performance of a vibration isolator using bellows and viscous fluid. Aerospace Science and Technology, Vol. 45, 2015, p. 1-9.

[3] Liu C., Jing X., Daley S., Li F. Recent advances in micro-vibration isolation. Mechanical System and Signal Processing, Vol. 56, Issue 1, 2015, p. 55-80.

[4] Wang P., Wang W., Ding J. W., Cheng Z. F., Liu J. Y. Vibration damping design for airborne electro-optical surveillance platform. Optical and Precision Engineering, Vol. 19, Issue 1, 2011, p. 83-89.

[5] Fu M., Liu Y., Cui M. L., Cao M. Metal-rubber vibration absorber for aerocraft. Optics and Precision Engineering, Vol. 21, Issue 5, 2013, p. 1174-1182.

[6] Klenke S. E., Baca T. Structural dynamics test simulation and optimization for aerospace components. Proceedings of the Second Test and Evaluation International Aerospace Forum, 1996.

[7] Geethamma V. G., Asaletha R., Kalarikkal N., Thomas S. Vibration and sound damping in polymers. Resonance, Vol. 19, Issue 9, 2014, p. 821-833.

[8] Zhao Yunfeng Development of High Performance Polymer Materials in Aerospace Industry. Academic Papers of Polymer Materials Science and Engineering, Chengdu, 2014. 
[9] Dall'Asta A., Ragni L. Nonlinear behavior of dynamic systems with high damping rubber devices. Engineering Structure, Vol. 30, Issue 12, 2008, p. 3610-3618.

[10] Nicholson D. W., Nelson N. W. Finite element analysis in design with rubber. Rubber Chemistry and Technology, Vol. 63, Issue 3, 1990, p. 368-406.

[11] Hughes T. J. R. The Finite Element Method-Linear Static and Dynamic Finite Element Analysis. Prentice Hall, New Jersey, 1987.

[12] Haiting C. L. Z. Numerical methods for analysing static characteristics of rubber isolator. Journal of Vibration and Shock, Vol 3, 2005, p. 36.

[13] Sjoberg M. On Dynamic Properties of Rubber Isolators. Department of Vehicle Engineering, Stockholm, 2002.

[14] Sjoberg M. Rubber isolators-measurements and modelling using fractional derivatives and friction. Society of Automotive Engineer, Vol. 1, 2000, p. 3518

[15] Ning Dayong, Gao Yunguo, Liu Xifeng Vibration isolation platform base on high accurate displacement sensors. Optics and Precision Engineering, Vol. 18, Issue 3, 2010, p. 646-652.

[16] Norwood C. J., Dickens J. D. The effect of vibration isolator properties and structural stiffness on isolator performance. Journal of Vibration and Control, Vol. 4, Issue 3, 1998, p. 253-275.

[17] Thomson D. J., Van Vliet W. J., Verheij J. W. Development of the indirect method for measuring the high frequency dynamic stiffness of resilient element. Sound and Vibration, Vol. 213, Issue 1, 1998, p. 169-188.

[18] Masterson R. A., Miller D. W., Grogan R. L. Development and validation of reaction wheel disturbance models: empirical model. Journal of Sound and Vibration, Vol. 249, Issue 3, 2002, p. $575-598$.

[19] Liu Jiayan, Cheng Zhifeng, Wang Ping Design and applications of rubber shock absorber in airborne photoelectric pod. China Mechanical Engineering, Vol. 12, Issue 10, 2014, p. 1308-1312.

[20] Zhu Shijian, et al. Vibration Theory and Vibration Isolation. National Defense Industry Press, Beijing, 2008.

[21] Hadden S., et al. Heavy load vibration isolation system for airborne pay-loads. Proceedings of SPIE, Vol. 4332, 2001, p. 171-182.

[22] Yang Hongtao, et al. The research of the high precision universal stable reconnaissance platform in near space. Proceedings of SPIE, Vol. 8196, 2011, p. 111-116.

[23] Lin T. R., Farag N. H., Pan J. Evaluation of frequency dependent rubber mount stiffness and damping by impact test. Applied Acoustics, Vol. 66, Issue 7, 2005, p. 829-844.

[24] Sjoberg M., Kari L. Testing of nonlinear interaction effects of sinusoidal and noise excitation on rubber isolation stiffness. Polymer Testing, Vol. 22, Issue 3, 2003, p. 343-351.

[25] Acoustics and Vibration - Laboratory Measurements of the Vibroacoustic Transfer Properties of Resilient Elements, ISO 10846.

[26] Sun Ningbo FOG Combination of Calibration and Error Compensation Technology Research. Master Thesis, Harbin Engineering University, 2013.

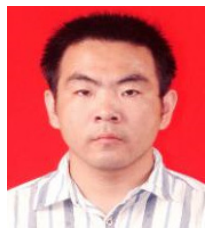

Changcheng Deng is a Ph.D. student at Changchun Institute of Optics, Fine Mechanics and Physics, Chinese Academy of Sciences. His current research interest is mechanical vibration of space remote sensors.

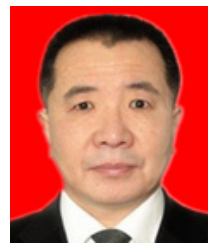

Deqiang Mu received his Ph.D. degree from Jilin University, Changchun, China, in 2001. Now he works at Changchun University of Technology. His current research interests include mechanical dynamics and optimization design, and measurement and control technologies for manufacturing processes. 
Yuan An received his Ph.D. degree from Changchun Institute of Optics, Fine Mechanics and Physics, Chinese Academy of Sciences, in 2007. Now he works at Chang Guang Satellite Technology Co., Ltd. His current interest is structure and mechanism design of space remote sensors.

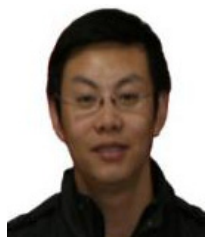

Yong Yan received his Ph.D. degree from Changchun Institute of Optics, Fine Mechanics and Physics, Chinese Academy of Sciences, in 2009. Now he works at Changchun Institute of Optics, Fine Mechanics and Physics. His current research interests include design and analysis of entire satellites, steadying of satellites to improve imaging, and integrated design and analysis of space optical loads.

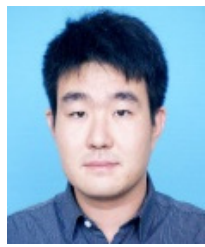

Zongxuan Li received his Ph.D. degree from Changchun Institute of Optics, Fine Mechanics and Physics, Chinese Academy of Sciences, in 2014. His current research interest is the integrated design and analysis of space optical remote sensors. 